\title{
THE LEVI PROBLEM ON STRONGLY PSEUDOCONVEX $G$-BUNDLES
}

\author{
JOE J PEREZ
}

\section{Abstract}

Let $G$ be a unimodular Lie group, $X$ a compact manifold with boundary, and $M$ the total space of a principal bundle $G \rightarrow M \rightarrow X$ so that $M$ is also a strongly pseudoconvex complex manifold. In this work, we show that if $G$ acts by holomorphic transformations in $M$, then the space of square-integrable holomorphic functions on $M$ is infinite $G$-dimensional. We also establish the following: Let $z$ be a point of the boundary $M$. Then there exists a holomorphic function with no smooth extension beyond $z$.

\section{INTRODUCTION}

Let $\bar{M}$ be a complex manifold with nonempty smooth boundary $b M, \bar{M}=$ $M \cup b M$, so that $M$ is the interior of $\bar{M}$, and $\operatorname{dim}_{\mathbb{C}}(M)=n$. Let us also assume for simplicity that $b M \subset \tilde{M}$ where $\tilde{M}$ is a complex neighborhood of $b M, \operatorname{dim}_{\mathbb{C}} \tilde{M}=n$, so that every point $z \in b M$ is an interior point of $\tilde{M}$. Also, choose a smooth function $\rho: \tilde{M} \rightarrow \mathbb{R}$ so that

$$
M=\{z \mid \rho(z)<0\}, \quad b M=\{z \mid \rho(z)=0\},
$$

and for all $z \in b M$, we have $d \rho(z) \neq 0$.

For any $z \in b M$ define the holomorphic tangent plane to the boundary by

$$
T_{z}^{\mathbb{C}}(b M)=\left\{w \in \mathbb{C}^{n} \mid \sum_{\nu=1}^{n} \frac{\partial \rho}{\partial z_{\nu}}(z) w_{\nu}=0\right\},
$$

and for $z \in b M$, define the quadratic form $L_{z}$ by

$$
L_{z}(w, \bar{w})=\sum_{\mu, \nu=1}^{n} \frac{\partial^{2} \rho}{\partial z_{\mu} \partial \bar{z}_{\nu}}(z) w_{\mu} \bar{w}_{\nu}, w \in T_{z}^{\mathbb{C}}(b M) .
$$

The complex manifold $M$ is said to be strongly pseudoconvex if for every $z \in b M$, the form $L_{z}$ is positive definite.

Denote by $\mathcal{O}(M)$ the set of all holomorphic functions on $M$. One can ask whether, for each point $z \in b M$, there exists an $f \in \mathcal{O}(M)$ which blows up at $z$. The origin of this problem is in the attempt to characterize domains of holomorphy, $[\mathrm{L}$. See [Si] for a review of the motivation and history of this problem. Let us say 
a point $z$ is peak point for $\mathcal{O}(M)$ if there exists an $f \in \mathcal{O}(M)$ unbounded on any neighborhood of $z$ and bounded in the complement of that neighborhood.

The Oka-Grauert theorem $\mathrm{Gr}$ ] asserts that if $\bar{M} \subset \mathbb{C}^{n}$ is compact, has nonempty boundary, and is strongly pseudoconvex, then every point of the boundary is a peak point for $\mathcal{O}(M)$. One way of proving this theorem and its variants will be described in this introduction.

A point $z \in b M$ is called a local peak point for $\mathcal{O}(M)$ if there exists a function $f \in \mathcal{O}(M)$ and a neighborhood $V$ of $z$ in $M$ such that $f$ is unbounded on $V$, but bounded on $V \backslash U_{z}$ for any neighborhood $U_{z}$ of $z$ in $M$. It was proven in GHS] that if $M$ is a strongly pseudoconvex complex manifold admitting a free cocompact holomorphic action of a discrete group, then every point in the boundary of $M$ is a local peak point for $\mathcal{O}(M)$.

The goal of this work is to establish the existence of nontrivial $L^{2}$ holomorphic functions on a strongly pseudoconvex manifold $M$, the total space of a principal $G$-bundle

$$
G \longrightarrow M \longrightarrow X
$$

with a compact base $X$, where we assume that the action of $G$ is holomorphic. We also establish that for any point $z \in b M$, there exists a holomorphic function for which a normal derivative of sufficiently high order is unbounded in any neighborhood of $z$.

We begin by reviewing the case in which $M$ is compact, FK. Suppose $M$ is a compact complex manifold whose boundary is strongly pseudoconvex and for a point $z_{0} \in b M$, we want a holomorphic function blowing up at $z_{0}$. Define the antiholomorphic exterior derivative $\bar{\partial}: \Lambda^{0,0} \rightarrow \Lambda^{0,1}$ in local coordinates $\left(z_{k}\right)$ by $\bar{\partial} u=\sum \frac{\partial u}{\partial \bar{z}_{k}} d \bar{z}_{k}$. If it can be established that

$$
\bar{\partial} u=\phi
$$

has a smooth solution $u$ whenever $\phi$ is a smooth antiholomorphic one-form that satisfies the compatibility condition $\bar{\partial} \phi=0$, then we may construct the function desired. The first step is to use Levi's construction to use the pseudoconvexity property of the boundary to define a function $f$, holomorphic in a neighborhood $U_{z_{0}}$ of $z_{0} \in b M$, that blows up just at $z_{0}$. Next, we can take a smooth function $\chi$ with support in $U_{z_{0}}$ that is identically equal 1 close to $z_{0}$. Extending $\chi f$ by zero we obtain a function defined everywhere and smooth away from $z_{0}$. Furthermore, $\bar{\partial} \chi f=0$ near $z_{0}$, so it can be extended smoothly to the boundary. If we can now find a smooth solution to $\bar{\partial} u=\bar{\partial} \chi f$, then $\chi f-u$ will be holomorphic and must blow up at $z_{0}$ since $u$ is smooth up to the boundary.

Let us describe the construction of solutions $u \in L^{2}(M)$ to $\bar{\partial} u=\phi$ with $\phi \in$ $L^{2}\left(M, \Lambda^{0,1}\right), \bar{\partial} \phi=0$. Note that solutions will only be determined modulo $\operatorname{Ker}(\bar{\partial}) \cap$ $L^{2}(M)$. As we prefer to deal with self-adjoint operators, we seek $u$ of the form 
$u=\bar{\partial}^{*} v$ satisfying

$$
\bar{\partial} \bar{\partial}^{*} v=\phi
$$

In order to do away with the compatibility condition on $\phi$ let us add a term $\bar{\partial}^{*} \bar{\partial} v$, obtaining

$$
\left(\bar{\partial} \bar{\partial}^{*}+\bar{\partial}^{*} \bar{\partial}\right) v=\phi .
$$

Define the operator $\square=\bar{\partial} \bar{\partial}^{*}+\bar{\partial}^{*} \bar{\partial}$. An easy computation shows that when $\bar{\partial} \phi=0$ is true, equation (2) reduces to equation (1).

To prove the solvability of the equation (2), we will see below, it suffices to prove that the operator $\square$ is Fredholm, i.e. the spaces Ker $\square$ and Coker $\square$ have finite dimensions.

The equation $\square u=\phi$ is a noncoercive boundary value problem. It has been shown $[\mathrm{K}, \mathrm{FK}, \mathrm{E}]$ that on its domain in the antiholomorphic $q$-forms, when $q>0$, the operator $\square+1$ has the following regularity property. Let $\zeta, \zeta_{1}$ be smooth cutoff functions for which $\zeta_{1}=1$ on $\operatorname{supp}(\zeta)$ and let $H^{s}\left(M, \Lambda^{0, q}\right)$ be the integer Sobolev space of sections in $\Lambda^{0, q}$ over $M$. Then $\square v+v \in H_{\text {loc }}^{s}\left(M, \Lambda^{0, q}\right)$ implies $v \in H_{\mathrm{loc}}^{s+1}\left(M, \Lambda^{0, q}\right)$ and there exist constants $C_{s}$ so that

$$
\|\zeta v\|_{H^{s+1}(M)} \leq C_{s}\left(\left\|\zeta_{1}(\square+1) v\right\|_{H^{s}(M)}+\|(\square+1) v\|_{L^{2}(M)}\right)
$$

uniformly in $v$.

These inequalities imply that the operator $(\square+1)^{-1}$ is bounded from $L^{2}\left(M, \Lambda^{0, q}\right)$ to $H^{1}\left(M, \Lambda^{0, q}\right)$ and so by Rellich's lemma is a compact operator in $L^{2}\left(M, \Lambda^{0, q}\right)$. Hence $\square$ has finite-dimensional kernel and cokernel and closed image (i.e. it is a Fredholm operator).

Now, one can solve equation (2) for all $\phi$ orthogonal to the finite-dimensional kernel. As $\chi f$ is unbounded, raising $f$ to arbitrarily high powers generates linearly independent functions, still holomorphic in a neighborhood of $z_{0}$. Further, since the $\chi f^{m}$ have compact support, $\bar{\partial}$ is injective on the vector space generated by $\left\{\chi f^{m} \mid m=1 \ldots N\right\}$. It follows that for $N$ sufficiently large,

$$
Q_{N}=\operatorname{Im}(\square) \cap \operatorname{span}_{\mathbb{C}}\left\{\bar{\partial} \chi f^{m} \mid m=1 \ldots N\right\} \neq\{0\} .
$$

Thus $\bar{\partial} \bar{\partial}^{*} u=\phi$ can be solved for $\phi \in Q_{N}$. Since all the forms $\bar{\partial} \chi f^{m}$ are smooth, this $\phi$ will be smooth and so we proceed as indicated above.

When $M$ is not compact, Rellich no longer applies, so the kernel and/or cokernel of $\square$ may be infinite-dimensional and the image of $\square$ may be not closed. In GHS], the von Neumann dimension of invariant subspaces of $L^{2}(\Gamma)$ is used in order to measure the kernel and cokernel of $\square$ in this setting as well as to measure the images of $\square$ 's spectral projections. We describe this briefly.

A closed subspace $L \subset L^{2}(\Gamma)$ is called invariant if it is invariant with respect to the right-translations $R_{\gamma}, \gamma \in \Gamma$. Defining $\mathcal{B}\left(L^{2}(\Gamma)\right)$ to be the bounded linear 
operators in $L^{2}(\Gamma)$ and the von Neumann algebra

$$
\mathcal{L}_{\Gamma}=\left\{L_{h} \mid h: \Gamma \rightarrow \mathbb{C} \text { and } L_{h} \in \mathcal{B}\left(L^{2}(\Gamma)\right)\right\}
$$

we have a trace on $\mathcal{L}_{\Gamma}$ defined by

$$
\operatorname{tr}_{\Gamma}\left(L_{h}\right)=h(e) .
$$

For an invariant subspace $L=\operatorname{Im}\left(L_{h}\right)$ with $L_{h}$ a self-adjoint projection, we define its $\Gamma$-dimension

$$
\operatorname{dim}_{\Gamma}(L)=\operatorname{tr}_{\Gamma}\left(L_{h}\right)=h(e)
$$

Note that $\operatorname{dim}_{\Gamma}\left(L^{2}(\Gamma)\right)=1$. Now, when $\Gamma$ acts freely on a manifold $M$ with quotient $X$, one can decompose the Hilbert space $L^{2}(M) \cong L^{2}(\Gamma) \otimes L^{2}(X)$ and define a trace

$$
\operatorname{Tr}_{\Gamma}=\operatorname{tr}_{\Gamma} \otimes \operatorname{Tr}_{\mathcal{B}\left(L^{2}(X)\right)}
$$

on the invariant operators $A \in \mathcal{B}\left(L^{2}(M)\right)^{\Gamma}$. It is with the corresponding dimension that closed, invariant subspaces of $L^{2}(M)$ are measured.

In GHS], it is shown that an a priori regularity estimate implies that $\square$ is $\Gamma$ Fredholm in the sense that $\operatorname{Im}(\square)$ contains a closed, $\Gamma$-invariant subspace of finite $\Gamma$-codimension. This, in turn, provides that the image of $\square$ intersected with the spaces

$$
L_{N}=L^{2}(\Gamma) \otimes \operatorname{span}_{\mathbb{C}}\left\{\bar{\partial} \chi f, \bar{\partial} \chi f^{2}, \ldots, \bar{\partial} \chi f^{N}\right\} \cong L^{2}(\Gamma) \otimes \mathbb{C}^{N} .
$$

contains closed, invariant subspaces of finite $\Gamma$-codimension in $L_{N}$. Because $\bar{\partial}$ is injective on these functions of small support, $\operatorname{dim}_{\Gamma}\left(L_{N}\right)=N$ and the intersection $\operatorname{Im}(\square) \cap L_{N} \subset L_{N}$ will be nontrivial if $N$ is sufficiently large. Picking a form $\phi \neq 0$ in the intersection, one sees that it is smooth so $\square u=\phi$ is solvable and the rest of the argument is as previously described.

In $[$ Per], the $\Gamma$-Fredholm property of $\square$ of [GHS] was adapted to the situation in which the discrete group $\Gamma$ is replaced by a unimodular Lie group $G$. For a unimodular group with its biinvariant measure fixed, the relevant von Neumann dimension is given by the $\operatorname{trace}^{\operatorname{tr}_{G}}$ on $\mathcal{L}_{G} \subset \mathcal{B}\left(L^{2}(G)\right)$ agreeing with

$$
\operatorname{tr}_{G}\left(L_{h}^{*} L_{h}\right)=\int_{G}|h(s)|^{2} d s
$$

whenever $L_{h} \in \mathcal{B}\left(L^{2}(G)\right)$ and $h \in L^{2}(G)$. The construction of the invariant trace $\operatorname{Tr}_{G}=\operatorname{tr}_{G} \otimes \operatorname{Tr}_{\mathcal{B}\left(L^{2}(X)\right)}$ will be described below.

The main result of $[\mathrm{Per}$ is the following: Assume that $G$ is a unimodular Lie group and $G \rightarrow \bar{M} \rightarrow X$ a principal $G$-bundle. Assume further that the total space $M$ is a strongly pseudoconvex complex manifold on which $G$ acts by holomorphic transformations and that $X$ is compact. Then, for $q>0$, the operator $\square$ in $\Lambda^{p, q}(M)$ is $G$-Fredholm.

The scope of the present paper is to continue in the direction set by GHS] described above and show 
Theorem 2.1. Assume that $G$ is a unimodular Lie group and $G \rightarrow M \rightarrow X$ a principal $G$-bundle. Assume further that the total space $M$ is a strongly pseudoconvex complex manifold on which $G$ acts by holomorphic transformations and that $X$ is compact. Then

$$
\operatorname{dim}_{G} L^{2} \mathcal{O}(M)=\infty
$$

and at each point $z \in b M$, there is an $f \in L^{2} \mathcal{O}(M)$ that is not smooth at $z$.

Remark 2.2. The result in GHS is easily extensible to the case of our $G$-bundles when $G$ has a discrete cocompact subgroup $\Gamma \subset G$. Generically, however, it is not the case that a unimodular Lie group have such a subgroup, $c f$. [M]. Examples of manifolds satisfying the hypotheses of the theorem are Grauert tubes of unimodular Lie groups. The unimodularity of $G$ is necessary for the definition of the $G$-Fredholm property. Recent work involving related questions can be found in $[\mathrm{Br}, \mathrm{TCM}]$.

Section 2 contains methods of measuring the $G$-dimensions of closed, invariant subspaces of $L^{2}(M)$. Section 3 describes the method of constructing large, smooth, invariant subspaces of $L^{2}(M)$ which we will use in order to solve the Levi problem. In section 4 we construct local expressions for functions that will be useful. In section 5 we prove that $\operatorname{dim}_{G} L^{2} \mathcal{O}(M)=\infty$. Section 6 discusses a method by which the problem may be adjusted so as to give holomorphic functions with stronger singularities.

\section{Paley-Wiener Theorems}

Definition 3.1. Let $M$ be a $G$-manifold with an invariant measure. For $f \in$ $L^{2}(M)$, define $\langle f\rangle \subset L^{2}(M)$ to be the $L^{2}$-closure of the complex vector space generated by right-translates of $f$ by $G$. In symbols,

$$
\langle f\rangle=\overline{\left\{\sum_{k}^{\text {finite }} \alpha_{k} f\left(\cdot t_{k}\right) \mid \alpha_{k} \in \mathbb{C}, t_{k} \in G\right\}}{ }^{L^{2}(M)} .
$$

This section is a small modification of a part of $\mathrm{AL}$.

Theorem 3.2. AL] Let $G$ be a locally compact unimodular group containing a closed, noncompact, connected set. Let $f$ be in $L^{2}(G)$ such that meas $(\operatorname{supp}(f))<$ meas $(G)$ and such that there exists $h$ in $L^{2}(G)$ with $L_{h} f=f$. Then $f=0$, m-a.e.

Corollary 3.3. If $0 \neq h \in L^{2}(M)$ has sufficiently small support, then $\operatorname{dim}_{G}\langle h\rangle=$ $\infty$.

Proof. Let the support of $h$ lie in a trivialization $G \times U, U \subset X$ of $M$ and choose a section so that we may write $h=h(t, x), t \in G, x \in X$. Also let $P$ be a self-adjoint invariant projection whose image contains $\langle h\rangle$. By invariance

$$
P R_{t} h=R_{t} h
$$


for any $t \in G$. By Lemma 1.2 of [AL], there exists a sequence $\left(t_{k}\right)_{k} \subset G$ for which the functions $\left(R_{t_{k}} h\right)_{k}$ are linearly independent and for which $S=\overline{\cup_{k} \operatorname{supp}\left(R_{t_{k}} h\right)}$ has finite measure. Denote by $\chi_{S}$ the characteristic function of $S$. The operator $u \mapsto \chi_{S} P u$ then has an infinite-dimensional eigenspace $\operatorname{span}\left\{R_{t_{k}} h \mid k \in \mathbb{N}\right\}$ corresponding to the eigenvalue one and so must not be compact.

We compute the Hilbert-Schmidt norm of the operator $\chi_{S} P$. Since $P$ is invariant, its distributional kernel takes the form

$$
(P u)(t, x)=\int_{G \times X} d s d y K(t, s ; x, y) u(s, y)=\int_{G \times X} d s d y \kappa\left(s t^{-1} ; x, y\right) u(s, y) .
$$

If $\left(\psi_{k}\right)_{k}$ is an orthonormal basis for $L^{2}(X)$, the Schwartz kernel of $\chi_{S} P$ can be expanded

$$
\chi_{S}(t) \kappa\left(s t^{-1} ; x, y\right)=\chi_{S}(t) \sum_{k l} H_{k l}\left(s t^{-1}\right) \psi_{k}(x) \bar{\psi}_{l}(y) .
$$

Since $\left(\psi_{k} \otimes \bar{\psi}_{l}\right)_{k l}$ forms an orthonormal basis for $L^{2}(X \times X), H_{k l}$ is equal the $k l^{\text {th }}$ Fourier coefficient of $\kappa$ with respect to the decomposition $L^{2}(G \times X \times X) \cong$ $\bigoplus_{k l}\left(L^{2}(G) \otimes \psi_{k} \otimes \bar{\psi}_{l}\right)$. We obtain

$$
\begin{gathered}
\left\|\chi_{S} P\right\|_{H S}^{2}=\int_{M \times M}\left|\chi_{S} K\right|^{2}=\int_{G \times G} d s d t\left|\chi_{S}(t)\right|^{2} \sum_{k l}\left|H_{k l}\left(s t^{-1}\right)\right|^{2} \\
=\sum_{k l}\left\|H_{k l}\right\|^{2} \int_{G} d t\left|\chi_{S}(t)\right|^{2}
\end{gathered}
$$

and conclude that $\sum_{k l}\left\|H_{k l}\right\|^{2}=+\infty$, for if not, we would have a Hilbert-Schmidt (and thus compact) operator $\chi_{S} P$ with an infinite-dimensional eigenspace corresponding to eigenvalue one.

We describe the invariant trace in $L^{2}(M)$, [T]. Again using the orthonormal basis $\left(\psi_{k}\right)_{k}$ of $L^{2}(X)$, we have

$$
L^{2}(M) \cong L^{2}(G) \otimes L^{2}(X) \cong \bigoplus_{k \in \mathbb{N}} L^{2}(G) \otimes \psi_{k}
$$

Denoting by $P_{k}$ the projection onto the $k^{\text {th }}$ summand in (5), we obtain a matrix representation of any operator $A \in \mathcal{B}\left(L^{2}(M)\right)$ with elements $A_{k l}=P_{k} A P_{l} \in$ $\mathcal{B}\left(L^{2}(G)\right)$. If $A \in \mathcal{B}\left(L^{2}(M)\right)^{G}$, we recover the $H_{k l}$ from above as matrix elements

$$
A \leftrightarrow\left[A_{k l}\right]_{k l}=\left[L_{H_{k l}}\right]_{k l} .
$$

The $G$-trace of such an operator is given by

$$
\operatorname{Tr}_{G}(A)=\sum_{k} \operatorname{tr}_{G}\left(L_{H_{k k}}\right)
$$


If $P$ is a self-adjoint projection, we compute $\operatorname{Tr}_{G}\left(P^{*} P\right)=\sum_{k l} \operatorname{tr}_{G}\left(L_{H_{k l}}^{*} L_{H_{k l}}\right)=$ $\sum_{k l}\left\|H_{k l}\right\|_{L^{2}(G)}^{2}$ by normality of $\operatorname{tr}_{G}$ and Equation (4). Thus $\operatorname{dim}_{G}\langle h\rangle=\operatorname{Tr}_{G}(P)=$ $\sum_{k l}\left\|H_{k l}\right\|^{2}=\infty$

\section{Smooth Invariant Closed Subspaces}

4.1. The group intrinsically. We gather some algebraic results. Define $\tilde{\alpha}(t)=$ $\alpha\left(t^{-1}\right)$ for any distribution $\alpha$ on $G$. The right-convolutions satisfy

$$
\left(R_{\alpha} \beta\right)(t) \stackrel{\text { def }}{=} \int_{G} d s \alpha(s) \beta(t s)=\int_{G} d s \beta(s) \alpha\left(t^{-1} s\right)=\left(R_{\beta} \alpha\right)\left(t^{-1}\right),
$$

so $R_{\alpha} \beta=\widetilde{R_{\beta} \alpha}$, and if $G$ is unimodular, then $\left\|R_{\alpha} \beta\right\|_{L^{2}(G)}=\left\|R_{\beta} \alpha\right\|_{L^{2}(G)}$. Using the definition $\left(L_{s} \alpha\right)(t)=\alpha\left(s^{-1} t\right)$, we obtain the identity

$$
\begin{aligned}
& \left(R_{\alpha} R_{\beta} \gamma\right)(t)=\int_{G} d s \alpha(s)\left[\int_{G} d r \beta(r) \gamma(t s r)\right] \\
= & \int_{G} d r\left[\int_{G} d s \alpha(s) \beta\left(s^{-1} r\right)\right] \gamma(t r)=\left(R_{\left[L_{\alpha} \beta\right]} \gamma\right)(t) .
\end{aligned}
$$

Assume $H \in C_{c}^{\infty}(G)$ and consider $\langle H\rangle \subset L^{2}(G)$ to be the $L^{2}$-closure of linear combinations of right-translations of $H$. Any $g \in\langle H\rangle$ satisfies $g=\lim _{m} g_{m}$ with $g_{m}=R_{\Delta_{m}} H$ for some sequence $\left(\Delta_{m}\right)_{m} \subset C_{c}^{\infty}(G)$. Equivalently, $\left(g_{m}\right)_{m}$ is Cauchy, thus

(6) $\left\|g_{m}-g_{n}\right\|=\left\|R_{\Delta_{m}} H-R_{\Delta_{n}} H\right\|=\left\|\left(R_{\Delta_{m}}-R_{\Delta_{n}}\right) H\right\|=\left\|R_{H}\left(\Delta_{m}-\Delta_{n}\right)\right\| \longrightarrow 0$.

Definition 4.1. Let $R_{H}=U\left|R_{H}\right|$ be the polar decomposition of $R_{H}$ and $\left|R_{H}\right|=$ $\int_{0}^{C} \lambda d E_{\lambda}$ be the spectral decomposition of $\left|R_{H}\right|$. For $\delta \in[0, C] \cup\left\{0^{+}\right\}$, let $P_{\delta}=$ $\int_{\delta}^{C} d E_{\lambda}$ and define

$$
\langle H\rangle_{\delta}=\left\{g \in\langle H\rangle \mid P_{\delta} U^{*} \tilde{g}=U^{*} \tilde{g}\right\} .
$$

Remark 4.2. Since $\operatorname{Im}\left(\left|R_{H}\right|\right)=\operatorname{Im}\left(R_{H}^{*} R_{H}\right) \subset C^{\infty}(G)$, we have $\operatorname{Im}\left(P_{\delta}\right) \subset C^{\infty}(G)$ for all $\delta \in(0, C]$.

Lemma 4.3. If $\delta>0$, then $g \in\langle H\rangle_{\delta}$ implies that $g=R_{\Delta} H$ for some $\Delta \in L^{2}(G)$. Consequently, $\langle H\rangle_{\delta} \subset L^{2} \cap C^{\infty}(G)$.

Proof. As in (6) , let $R_{\Delta_{m}} H \rightarrow g \in\langle H\rangle_{\delta}$. Then $R_{H} \Delta_{m} \rightarrow \tilde{g}$ and

$$
U P_{\delta} U^{*} R_{H} \Delta_{m} \rightarrow U P_{\delta} U^{*} \tilde{g}=\tilde{g} .
$$

The composition $P_{\delta} U^{*} R_{H}=P_{\delta}\left|R_{H}\right|=P_{\delta}\left|R_{H}\right| P_{\delta}$, when restricted to the orthogonal complement of $\operatorname{Ker}\left(P_{\delta}\right)$, is an injection with bounded inverse, as is $U P_{\delta} U^{*} R_{H}$. Therefore there exists a Cauchy sequence $\left(\Delta_{m}^{\prime}\right)_{m}$ in $L^{2}(G) \ominus \operatorname{Ker}\left(P_{\delta}\right)$ with limit $\Delta^{g} \in L^{2}(G) \ominus \operatorname{Ker}\left(P_{\delta}\right)$ so that

$$
g=R_{\Delta g} H
$$


Noting that $\Delta^{g} \in L^{2}(G)$ for all $g \in\langle H\rangle_{\delta}$ and $H \in C_{c}^{\infty}(G)$, we have $\langle H\rangle_{\delta} \subset$ $C^{\infty}(G)$.

The previous lemma gives that, if $\delta>0$, then $\langle H\rangle_{\delta} \subset\left\{R_{\Delta} H \mid \Delta \in \operatorname{Im}\left(P_{\delta}\right)\right\}$. In fact, the spaces are equal:

Lemma 4.4. Let $\left|R_{H}\right|=\int_{0}^{C} \lambda d E_{\lambda}$ and $P_{\delta}=\int_{\delta}^{C} d E_{\lambda}$ as before. Then, for any $\delta>0$, we have $\langle H\rangle_{\delta}=\left\{R_{\Delta} H \mid \Delta \in \operatorname{Im}\left(P_{\delta}\right)\right\}$.

Proof. For $\delta>0$, all $g \in\langle H\rangle_{\delta}$ satisfy

$$
\tilde{g}=U P_{\delta} U^{*} \tilde{g}=U P_{\delta} U^{*} R_{H} \Delta^{g}=U P_{\delta}\left|R_{H}\right| \Delta^{g}=U\left|R_{H}\right| P_{\delta} \Delta^{g}=R_{H} P_{\delta} \Delta^{g},
$$

so each $g \in\langle H\rangle_{\delta}$ is of the form $R_{\Delta^{g}} H$ for $\Delta^{g} \in \operatorname{Im}\left(P_{\delta}\right)$. Conversely, if $\tilde{g}=R_{H} P_{\delta} \Delta^{g}$ for $\Delta^{g} \in \operatorname{Im}\left(P_{\delta}\right)$, the above chain of equalities can be read right to left, obtaining $\tilde{g}=R_{H} P_{\delta} \Delta^{g}=U P_{\delta} U^{*} \tilde{g}$.

Theorem 4.5. For $\delta \in(0, C]$, the spaces $\langle H\rangle_{\delta} \subset\langle H\rangle$ are closed, smooth, rightinvariant, and $\operatorname{dim}_{G}\langle H\rangle_{\delta} \rightarrow \infty$ as $\delta \rightarrow 0^{+}$.

Proof. The invariance condition on $\langle H\rangle_{\delta}$ is equivalent to the statement $g=R_{\Delta} H$ for $\Delta \in \operatorname{Im}\left(P_{\delta}\right)$ if and only if

$$
R_{t} g=R_{t} R_{\Delta} H=R_{\left[L_{t} \Delta\right]} H \in\langle H\rangle_{\delta} \quad(t \in G) .
$$

Since $P_{\delta}$ is a function of $R_{H}$, it commutes with all left-translations so $L_{t} \Delta \in$ $\operatorname{Im}\left(P_{\delta}\right)$.

For $\delta=0^{+}$, the condition

$$
P_{0^{+}} U^{*} \tilde{g}=U^{*} \tilde{g} \Leftrightarrow P_{0^{+}} U^{*} R_{H} \Delta=U^{*} R_{H} \Delta \Leftrightarrow P_{0^{+}}\left|R_{H}\right| \Delta=\left|R_{H}\right| \Delta
$$

defining $\langle H\rangle_{0^{+}}$is vacuous, so $\langle H\rangle_{0^{+}}=\langle H\rangle$. By Corollary [3.3, $\operatorname{dim}_{G}\left(\langle H\rangle_{0^{+}}\right)=\infty$. Since $\langle H\rangle_{\delta} \nearrow\langle H\rangle_{0^{+}}$, normality of the trace gives the result.

4.2. Actions. For a function $h \in C_{c}^{\infty}(M)$ with small enough support, we may choose a section and write $h$ as a smooth function of $(t, x) \in G \times U$ where $U \subset X$. Since $M$ has a global right $G$-action, we may write a convolution by $\Delta, R_{\Delta} \otimes 1_{L^{2}(X)}$, simply $R_{\Delta}$. We obtain an expression for $\left\|R_{\Delta} h\right\|_{L^{2}(M)}$ by first decomposing $h$ as in Equation (5). With $H_{k}(t)=\left\langle h(t, \cdot), \psi_{k}\right\rangle_{L^{2}(X)}$, the function $h=\sum_{k} H_{k} \otimes \psi_{k}$ and

$$
R_{\Delta} h=\sum_{k}\left(R_{\Delta} H_{k}\right) \otimes \psi_{k} \quad \text { so } \quad\left\|R_{\Delta} h\right\|_{L^{2}(M)}^{2}=\sum_{k}\left\|R_{\Delta} H_{k}\right\|_{L^{2}(G)}^{2} .
$$

Remark 4.6. Let $\delta>0$ and consider the decompositions $R_{H_{k}}=U_{k}\left|R_{H_{k}}\right|,\left|R_{H_{k}}\right|=$ $\int_{\delta}^{C} \lambda d E_{\lambda}^{k}$ and the projections $P_{\delta}^{k}=\int_{\delta}^{C} d E_{\lambda}^{k}$. Then, for each $l \in \mathbb{N}$ for which $R_{\Delta} H_{l} \neq 0$ we have

$$
\left\|R_{\Delta} h\right\|_{L^{2}(M)}^{2}=\sum_{k}\left\|R_{\Delta} H_{k}\right\|_{L^{2}(G)}^{2} \geq\left\|R_{\Delta} H_{l}\right\|_{L^{2}(G)}^{2} \geq \delta^{2}\|\Delta\|_{L^{2}(G)}^{2} \quad\left(\Delta \in \operatorname{Im}\left(P_{\delta}^{l}\right)\right) .
$$


This implies that $\operatorname{Im}\left(P_{\delta}^{l}\right) \ni \Delta \mapsto R_{\Delta} h$ is boundedly invertible as long as $R_{\Delta} H_{l} \neq 0$. Let us then take $\mathfrak{D}_{\delta}^{l}=\operatorname{Im}\left(P_{\delta}^{l}\right)$ for $R_{\Delta} H_{l} \neq 0$ and define

$$
\langle h\rangle_{\delta, l}=\left\{R_{\Delta} h \mid \Delta \in \mathfrak{D}_{\delta}^{l}\right\} .
$$

Lemma 4.7. For $\delta>0$, the spaces $\langle h\rangle_{\delta, l}$ are closed, invariant, and smooth. Furthermore, $\operatorname{dim}_{G}\langle h\rangle_{\delta}<\infty$.

Proof. The previous remark and Lemma 4.4 give that the space $\langle h\rangle_{\delta, l}$ is closed. For $\delta>0$, Lemma 4.4 also provides that $\mathfrak{D}_{\delta}^{l} \subset C^{\infty} \cap L^{2}(G)$, so, since all derivatives of $h$ are in $L^{1}(G)$ and

$$
\begin{aligned}
\left\|R_{\Delta} h\right\|_{L^{2}(M)}^{2} & =\int_{X} d x \int_{G} d t\left|\int_{G} d s \Delta(s) h(t s, x)\right|^{2} \leq\|\Delta\|_{L^{2}(G)}^{2} \int_{X} d x\|h(\cdot, x)\|_{L^{1}(G)}^{2} \\
& \lesssim\|\Delta\|_{L^{2}(G)}^{2}\left|\int_{X} d x\|h(\cdot, x)\|_{L^{1}(G)}\right|^{2}=\|\Delta\|_{L^{2}(G)}^{2}\|h\|_{L^{1}(M)}^{2},
\end{aligned}
$$

we have $\langle h\rangle_{\delta, l} \subset H^{\infty}(\bar{M})$. Corollary 6.4 of $[\overline{P e r}]$ states that if a space is closed, invariant, and in $H^{\infty}(\bar{M})$, then it has finite $G$-dimension.

Lemma 4.8. As $\delta \rightarrow 0^{+}, \operatorname{dim}_{G}\left(\langle h\rangle_{\delta, l}\right) \longrightarrow+\infty$.

Proof. By Equation (7), the space $\langle h\rangle_{\delta, l}$ has an orthogonal decomposition

$$
\langle h\rangle_{\delta, l}=\bigoplus_{k}\left\{R_{\Delta} H_{k} \mid \Delta \in \mathfrak{D}_{\delta}^{l}\right\} \otimes \psi_{k} .
$$

Now, since $H_{l}(t)=\left\langle h(t, \cdot), \psi_{l}\right\rangle_{L^{2}(X)} \in C_{c}^{\infty}(G)$, Theorem 4.5 holds and provides that

$$
\left\langle H_{l}\right\rangle_{\delta} \otimes \psi_{l}=\left\{R_{\Delta} H_{l} \mid \Delta \in \mathfrak{D}_{\delta}^{l}\right\} \otimes \psi_{l}
$$

is a closed, invariant subspace of $\langle h\rangle_{\delta, l}$ whose $G$-dimension is unbounded as $\delta \rightarrow$ $0^{+}$.

\section{Normal Coordinates and Singular Functions}

Following [FS], Section 18, we choose normal coordinates $\left(z_{k}\right)_{k=0}^{n}$ in a neighborhood of a point $\xi \in b M$. These have the following properties:

1) $\xi$ is an isolated point in the set $b M \cap\left\{\eta \mid z_{0}(\eta)=0\right\}$

2) There are functions $z_{k}=x_{k}+i y_{k}$ and $t=\mathfrak{R e} z_{0}$, for which $x_{1}, \ldots, x_{n}$, $y_{1}, \ldots, y_{n}, t$ form a coordinate system for a neighborhood of $\xi$ in $b M$. Furthermore, the defining function $\rho$ satisfies

$$
\rho(z)=-\mathfrak{I m} z_{0}+\|z\|^{2}+\mathcal{O}\left(\left|z_{0}\right|\|z\|+\|z\|^{3}\right)
$$

with $\|z\|^{2}=\sum_{1}^{n}\left|z_{k}\right|^{2}$. We will use the following notation. If $A$ and $B$ are functions on a set $S$, then we use the notation $A \lesssim B$ to mean that for some $C>0$, 
$A(s) \leq C B(s)$ for $s \in S$. Further we will write $A \approx B$ to mean that $A \lesssim B$ and $B \lesssim A$.

Now, since $\rho<0$ in $M$, Equation (9) gives that $\mathfrak{I m} z_{0}>0$ for $z$ sufficiently near $\xi$. Thus we may choose a branch of $\log z_{0}$ and define all real powers of $z_{0}$. We then define the Levi function

$$
f_{\tau}(z)=1 / z_{0}^{\tau} .
$$

Lemma 5.1. Define $|z|^{2}=\sum_{0}^{n}\left|z_{k}\right|^{2}$. Then for $z$ sufficiently near 0 we have

$$
|z|^{2} \lesssim\left|z_{0}\right| \leq|z|
$$

Proof. This is because

$$
\left|z_{0}\right| \geq \mathfrak{I m}\left(z_{0}\right) \geq \mathfrak{I m}\left(z_{0}\right)+\rho(z)=\|z\|^{2}+\mathcal{O}\left(\left|z_{0}\right|\|z\|+\|z\|^{3}\right)
$$

SO

$$
2\left|z_{0}\right| \geq\|z\|^{2}+\left|z_{0}\right|+\mathcal{O}\left(\left|z_{0}\right|\|z\|+\|z\|^{3}\right) \geq|z|^{2}+\mathcal{O}\left(\left|z_{0}\right|\|z\|+\|z\|^{3}\right) .
$$

Now for $\|z\|$ sufficiently small, the $\mathcal{O}\left(\left|z_{0}\right|\|z\|\right)$ term can be brought to the left side, obtaining

$$
\left|z_{0}\right| \gtrsim|z|^{2}+\mathcal{O}\left(\|z\|^{3}\right) \gtrsim|z|^{2}
$$

Lemma 5.2. For $\tau$ positive, $\chi f_{\tau} \in L^{1} \cap L^{2}(M)$ whenever $\tau<\frac{1}{2} \operatorname{dim}_{\mathbb{C}} M$.

Proof. Put $m=\operatorname{dim}_{\mathbb{C}} M$. For $\chi f_{\tau} \in L^{2}$ we only need $f_{\tau} \in L_{l o c}^{2}$. By (10), with $r=|z|$, we have

$$
\int_{B_{\epsilon}}\left|f_{\tau}\right|^{2} d V \lesssim \int_{0}^{\epsilon} \frac{r^{2 m-1}}{r^{4 \tau}} d r<\infty
$$

whenever $\tau<m / 2$. Similarly we get $f_{\tau} \in L_{l o c}^{1}$ for $\tau<m$.

Lemma 5.3. If $\tau$ is positive, $\delta>0$ and $k \geq \operatorname{dim}_{\mathbb{R}}(G)-\tau$, then $\left\langle\chi f_{\tau}\right\rangle_{\delta} \cap C^{k}(\bar{M})=$ $\{0\}$.

Proof. Since the support of $\chi$ is as small as we please, we may work locally: Near the point $\xi \in b M$ we have coordinates $x_{1} \ldots x_{n}, y_{1} \ldots y_{n}, t=\mathfrak{R e}\left(z_{0}\right)$ of the boundary and $\rho \approx-\mathfrak{I m}\left(z_{0}\right)$ as in Equation (9). Since $z_{0}=t-i \rho$ has an isolated zero at $\xi$, we have that

$$
z_{0}(\xi g)=t(\xi g)-i \rho(\xi g) \neq 0
$$

for any nonidentity group element $g$. But since the group action preserves the boundary, $\rho(\xi g)=0$ for any $g \in G$. Thus

$$
t(\xi g) \neq 0 \quad \text { whenever } \quad g \neq e .
$$

Now, if $\operatorname{dim}_{\mathbb{R}} G>1$, the set $\left\{t(\xi g) \mid g \in U_{e}\right\} \subset \mathbb{R}$ must not change signs. Otherwise, the intermediate value theorem would contradict $z_{0}$ 's having an isolated 
zero at $\xi$ (make a small loop about $\xi$ with the group). Assume without loss of generality that $t(\xi g) \geq 0$. In terms of the action, the inequality (10) reads

$$
|z(\xi g)|^{2} \lesssim\left|z_{0}(\xi g)\right|=t(\xi g) \lesssim|z(\xi g)|
$$

Since all norms in finite-dimensional spaces are equivalent, the group action is free, and the exponential function is a diffeomorphism in a neighborhood of the identity, it can be taken to be an isometry. Thus if $g=\exp (X)$, we have $|z(\xi g)|=$ $|\xi \exp (X)| \approx|X|$ for $X \in T_{e} G$ sufficiently small. Together,

$$
|X|^{2}=|\xi \exp (X)|^{2} \lesssim\left|(\xi \exp (X))_{0}\right| \lesssim|\xi \exp (X)|=|X|
$$

So

$$
|X|^{2} \lesssim t(\xi g) \lesssim|X|
$$

If we put $\operatorname{dim}_{\mathbb{R}}(G)=\nu, \mathfrak{B}=\left\{X \in T_{e} G|| X \mid \leq \epsilon\right\}$, and $B=\exp \mathfrak{B}$, we have

$$
\int_{0}^{\epsilon} \frac{r^{\nu-1} d r}{r^{\tau}} \approx \int_{\mathfrak{B}} \frac{d^{\nu} X}{|X|^{\tau}} \lesssim \int_{B} \frac{d g}{[t(\xi g)]^{\tau}}=\int_{B} \frac{d g}{\left[z_{0}(\xi g)\right]^{\tau}}=\int_{B} d g f_{\tau}(\xi g) .
$$

The first integral diverges for $\tau \geq \operatorname{dim}_{\mathbb{R}}(G)$. Now, for $\tau>0$

$$
\frac{\partial^{m}}{\partial \rho^{m}} f_{\tau}(z)=\frac{\partial^{m}}{\partial \rho^{m}} \frac{1}{(t-i \rho)^{\tau}} \approx \frac{1}{(t-i \rho)^{\tau+m}}=f_{\tau+m}(z)
$$

so, differentiating under the integral,

$$
\frac{\partial^{m}}{\partial \rho^{m}} \int_{B} d g f_{\tau}(\xi g) \approx \int_{B} d g f_{m+\tau}(\xi g)
$$

shows that smooth convolutions of $f_{\tau}$ are not smoothly differentiable $m \geq \operatorname{dim}_{\mathbb{R}}(G)-$ $\tau$ times at the boundary.

\section{Main Theorem}

In the notation of the previous sections, define

$$
\langle\langle\chi f\rangle\rangle_{\delta}=\left\{R_{\Delta} \chi f \mid \Delta \in \mathfrak{D}_{\delta}^{\bar{\partial} \chi f}\right\} .
$$

Since $\mathfrak{D}_{\delta}^{\bar{\partial} \chi f} \subset L^{2}(G)$, Equation 8 and Lemma 5.2 imply that in choosing $f=f_{\tau}$ with $\tau<\frac{1}{2} \operatorname{dim}_{\mathbb{C}}(M)$, we obtain $\langle\langle\chi f\rangle\rangle_{\delta} \subset L^{2}(M)$. Furthermore, $\chi f \in L^{2}(M)$ is in the domain of the Hilbert space operator $\bar{\partial}_{\mathcal{H}}$. Lemma 5.3 guarantees that $\langle\langle\chi f\rangle\rangle_{\delta} \cap C^{\infty}(\bar{M})=\{0\}$.

Lemma 6.1. The restricted antiholomorphic exterior derivative $\bar{\partial}:\langle\langle\chi f\rangle\rangle_{\delta} \rightarrow$ $\langle\bar{\partial} \chi f\rangle_{\delta}$ is a bijection.

Proof. The kernel is trivial because of the small $X$-support of the members of $\langle\langle\chi f\rangle\rangle_{\delta}$. Since $R_{\Delta} \chi f$ is smooth in the interior of $M$ for $\Delta \in \mathfrak{D}_{\delta}^{\bar{\partial} \chi f}, \bar{\partial} R_{\Delta} \chi f=$ $R_{\Delta} \bar{\partial} \chi f$. Since $\langle\langle\chi f\rangle\rangle_{\delta} \subset L^{2}(M), \bar{\partial}$ and $\bar{\partial}_{\mathcal{H}}$ coincide there. 
Theorem 6.2. The space $L^{2} \cap \mathcal{O}(M)$ is infinite-G-dimensional and for every point $z$ of the boundary of $M$, there exists a holomorphic function $\Phi_{z}$ which cannot be holomorphically extended beyond $z$.

Proof. Theorem 6.6 of $[\mathrm{Per}]$ provides that the operator $\square$ on its domain is $G$ Fredholm. Lemma 4.8 allows us to conclude that, for $\delta>0$ sufficiently small, there exist closed, invariant subspaces

$$
L_{\delta} \subset\langle\bar{\partial} \chi f\rangle_{\delta} \cap \operatorname{Im}(\square)
$$

of arbitrarily large $G$-dimension. Now for $\delta>0$ sufficiently small, let $0 \neq g \in L_{\delta}$ and solve

$$
\square u=g .
$$

By Lemma 4.7, $\langle\bar{\partial} \chi f\rangle_{\delta} \subset C^{\infty}\left(\bar{M}, \Lambda^{0,1}\right)$, so $g$ is smooth. The regularity of $\square$ then gives that $u \in C^{\infty}(\bar{M})$. Since the images of $\bar{\partial}$ and $\bar{\partial}^{*}$ are orthogonal, we have

$$
\square u=\bar{\partial} \bar{\partial}^{*} u=g
$$

and $g=\bar{\partial} \phi$ for some $\phi \in\langle\langle\chi f\rangle\rangle_{\delta}$ by Lemma 6.1. Form the holomorphic function

$$
\Phi=\phi-\bar{\partial}^{*} u \text {. }
$$

By Lemma 5.3, $\langle\chi f\rangle_{\delta} \cap C^{\infty}(\bar{M})=\{0\}$, from which $\phi \notin C^{\infty}(\bar{M})$. We conclude that $\Phi \notin C^{\infty}(\bar{M})$ and thus is nonzero.

Remark 6.3. The method is equivalent to mollifying the equation $\square u=\bar{\partial} \chi f$ with a group convolution $R_{\Delta} \square u=R_{\Delta} \square u=\bar{\partial} \chi f, \Delta \in C^{\infty}(G)$, using invariance.

\section{EXTENSIONS}

To obtain holomorphic functions with the strongest possible singularities at the boundary, it is in our interest while solving the $\bar{\partial}$-Neumann problem on $M$ to choose the symmetry group as small as possible. To this end we note that if $H \subset G$ then $\square$ is $H$-invariant too, but not necessarily $H$-Fredholm unless $G / H$ is compact. In this case we may profit by working the $H \rightarrow M \rightarrow X \times(G / H)$ problem instead of $G \rightarrow M \rightarrow X$, with $H$ having no noncompact subgroups $K \subset H$ giving rise to compact quotients. If $K$ is a maximal compact subgroup in $G$ then $H$ should be taken to be $K \backslash G$. The complex structure will be $H$-invariant and $H$ will again be unimodular by Theorem 8.36 of [ $\mathrm{Kn}]$.

\section{ACKnowledgments}

The author wishes to thank Gerald Folland and Jonathan Rosenberg for phone and email conversations and Mikhail Shubin for the problem and many years of friendship and mathematical help. 


\section{REFERENCES}

[AL] Arnal, D.; Ludwig, J.: Q.U.P. and Paley-Wiener Properties of Unimodular, Especially Nilpotent, Lie Groups, Proceedings of the AMS, 125, no 4, April 1997, 1071-1080

[Br] Brudnyi, A.: On Holomorphic $L^{2}$ functions on Coverings of Strongly Pseudoconvex Manifolds, arxiv.org/pdf/math.CV/0508237

[E] Engliš, M.: Pseudolocal Estimates for $\bar{\partial}$ on General Pseudoconvex Domains, Indiana Univ. Math. J., 50, (2001) no 4. 1593-1607, and Erratum, to appear in Indiana Univ. Math. J.

[FK] Folland, G. B. \& Kohn J. J.: The Neumann Problem for the Cauchy-Riemann Complex, Ann Math Studies, No. 75. Princeton University Press, Princeton, N.J. 1972

[FS] Folland, G.B. \& Stein,E., Estimates for the $\bar{\partial}_{b}$-complex and analysis on the Heisenberg group, C.P.A.M. 27 (1974), 429-522.

[Gr] Grauert, H.: On Levi's Problem and the Imbedding of Real-Analytic Manifolds, Ann. of Math., 68, (1958), 460-472

[GHS] Gromov, M., Henkin, G. \& Shubin, M.: Holomorphic $L^{2}$ Functions on Coverings of Pseudoconvex Manifolds, Geom. Funct. Anal., v. 8, no. 3, (1998), 552-585

[K] Kohn, J. J.: Harmonic Integrals on Strongly Pseudoconvex Manifolds, I \& II, Ann of Math, 78 (1963) 112-148 \& $\mathbf{7 9}$ (1964) 450-472

[Kn] Knapp, A. W.: Lie Groups Beyond an Introduction 2 ed., Birkhäuser, Boston, 2002

[L] Levi, E.E.: Studii sui punti singolari essenziali delle funzioni analitiche di due o più variabili complesse, Ann. Mat. Pura Appl. 17 (1910), 61-87

[M] Margulis, G.A.: Discrete Subgroups of Semisimple Lie Groups, Ergebnisse der Mathematik und ihrer Grenzgebiete, 17 Springer-Verlag, Berlin, 1991

[Per] Perez, J.J.: The G-Fredholm Property for the $\bar{\partial}$-Neumann Problem, Wherever it ends up

[Si] Siu, Y.-T.: Pseudoconvexity and the problem of Levi. Bulletin of the AMS, Volume 84 Number 4, 481-512, July 1978

[T] Takesaki, M.: Theory of Operator Algebras vol I, Springer-Verlag, Berlin, 1979

[TCM] Todor, R., Chiose, I., Marinescu, G.: $L^{2}$-Riemann-Roch Inequalities for Covering Manifolds, arxiv.org/pdf/math.AG/0002049 